\title{
Psoas Morphology Differs between Supine and Sitting Magnetic Resonance Imaging Lumbar Spine: Implications for Lateral Lumbar Interbody Fusion
}

\author{
Aaron J. Buckland, Bryan M. Beaubrun, Evan Isaacs, John Moon, Peter Zhou, Sam Horn, Gregory Poorman, \\ Jared C. Tishelman, Louis M. Day, Thomas J. Errico, Peter G. Passias, Themistocles Protopsaltis
}

Department of Orthopaedic Surgery, NYU Hospital for Joint Diseases, New York, NY, USA

\begin{abstract}
Study Design: Retrospective radiological review.
Purpose: To quantify the effect of sitting vs supine lumbar spine magnetic resonance imaging (MRI) and change in anterior displacement of the psoas muscle from $L 1-L 2$ to $L 4-L 5$ discs.

Overview of Literature: Controversy exists in determining patient suitability for lateral lumbar interbody fusion (LLIF) based on psoas morphology. The effect of posture on psoas morphology has not previously been studied; however, lumbar MRI may be performed in sitting or supine positions.

Methods: A retrospective review of a single-spine practice over 6 months was performed, identifying patients aged between 18-90 years with degenerative spinal pathologies and lumbar MRIs were evaluated. Previous lumbar fusion, scoliosis, neuromuscular disease, skeletal immaturity, or intrinsic abnormalities of the psoas muscle were excluded. The anteroposterior (AP) dimension of the psoas muscle and intervertebral disc were measured at each intervertebral disc from $L 1-L 2$ to $L 4-L 5$, and the AP psoas:disc ratio calculated. The morphology was compared between patients undergoing sitting and/or supine MRI.

Results: Two hundred and nine patients were identified with supine-, and 60 patients with sitting-MRls, of which 13 patients had undergone both sitting and supine MRIs (BOTH group). A propensity score match (PSM) was performed for patients undergoing either supine or sitting MRI to match for age, BMI, and gender to produce two groups of 43 patients. In the BOTH and PSM group, sitting MRI displayed significantly higher AP psoas:disc ratio compared with supine MRI at all intervertebral levels except L1-L2. The largest difference observed was a mean $32 \%-37 \%$ increase in sitting AP psoas:disc ratio at the $L 4-L 5$ disc in sitting compared to supine in the BOTH group (range, $0 \%-137 \%$ ).

Conclusions: The psoas muscle and the lumbar plexus become anteriorly displaced in sitting MRIs, with a greater effect noted at caudal intervertebral discs. This may have implications in selecting suitability for LLIF, and intra-operative patient positioning.
\end{abstract}

Keywords: Psoas; Intervertebral disc; Spinal fusion; Magnetic resonance imaging; Lumbosacral plexus

\section{Introduction}

The iliopsoas muscle functions as the primary flexor of the hip joint but whether it has any functional role with regard to the lumbar spine remains to be determined [1]. However, the iliopsoas is the only muscle group in the

Received Apr 19, 2017; Revised May 18, 2017; Accepted May 29, 2017

Corresponding author: Aaron J. Buckland

Department of Orthopaedic Surgery, NYU Hospital for Joint Diseases, 306 East 15th Street, New York, NY 10003, USA

Tel: +1-646-794-8640, Fax: +1-646-602-6927, E-mail: Aaron.Buckland@nyumc.org 
body that directly attaches to the spine, the pelvis and the femur, which implicates its importance and influence on spine and hip movement [2]. More importantly, located within the psoas muscle is the lumbar plexus, which is composed of the anterior rami of the L1, L2, and L3 nerve roots, part of the L4 anterior ramus and contributions from the subcostal nerve from $\mathrm{T} 12$, on the posterior abdominal wall $[3,4]$. This neural plexus presents a challenge to any surgical procedure that involves penetrating or retracting the psoas muscle.

Lateral lumbar interbody fusion (LLIF) has been advocated by some surgeons as a minimally invasive approach to reduce blood loss, minimize tissue damage and provide faster patient recovery $[5,6]$. This approach is based on the trans-psoas [6] or anterior-to-psoas $[5,7,8]$ technique and allows for direct lateral access to the intervertebral disc space [6]. However, the trans-psoas approach only allows access from L1-L2 to L4-L5 levels [4]. Due to variations in psoas anatomy and lumbar plexus, controversy exists in determining the safest approach techniques for LLIF to reduce the risk of injuring the lumbar plexus, particularly the L3 and L4 nerve root at the L4-L5 intervertebral disc $[4,9]$. When penetrating and dilating the psoas muscle, excessive neural retraction and psoas trauma, may cause post-operative thigh pain, numbness or quadriceps weakness, despite the use of electromyography (EMG) neuromonitoring [10]. To minimize the likelihood of lumbar plexus injury, preoperative assessment of the psoas anatomy and neuro-monitoring of the lumbar plexus is required for safe lateral approach to the lumbar spine.

Most lumbar magnetic resonance imaging (MRI) is performed in the supine position with the hips in a neutral position. However, some patients may present with sitting MRIs depending on patient claustrophobia or radiology facility marketing. Most surgeons will slightly flex the hip during the LLIF procedure to reduce tension on the psoas muscle, a position that would situate the psoas somewhere between the traditional sitting MRI and the alternative sitting open MRI. However, the influence of posture on the position of the psoas muscle and the lumbar plexus has not been previously reported. This study hypothesizes that psoas anatomy and the position of the lumbar plexus will vary between sitting and supine postures which will have implications in determining preoperative suitability for LLIF. In particular, postural differences in anatomy will be more variable at more caudal intervertebral discs.

\section{Materials and Methods}

\section{Patient selection}

A retrospective review was conducted of a single spine surgery practice over a 6-month period in order to identify patients who had undergone an MRI of the lumbar spine for evaluation of degenerative spinal pathologies. Inclusion criteria were adult patients between 18-90 years of age, with degenerative lumbar spinal pathology presenting between 2015-2016. Patients were excluded if they had scoliosis, neuromuscular disease, previous lumbar fusion, previous abdominal infection, or had intrinsic abnormalities of the psoas muscle (e.g., tumor, infection, or trauma). Institutional Review Board approval was obtained by ethics review board (NYU Langone Health; IRB approval no., S16-01429).

\section{Radiographic measurement}

MRI image acquisition was performed with either a $1.5 \mathrm{~T}$ or 3T magnet, in a sitting or supine position. The postural position was not randomized. Axial T1 images were analyzed at each intervertebral level to assess the morphology of the psoas muscle at reach intervertebral level. The anteroposterior (AP) dimension of the psoas muscle was measured at each disc space of both the right and left psoas muscle from L1-L2, L2-L3, L3-L4, and L4-L5 and compared to the AP dimension of the intervertebral disc, as measured by the AP length of the superior endplate of the caudal vertebra (Fig. 1). The AP psoas:disc ratio was then calculated and compared between patients undergoing sitting and/or supine lumbar spine MRIs.

\section{Statistical analysis}

The aim of the study was to use the ratio between the AP psoas muscle and the intervertebral disc to determine a $\%$ increase or decrease in that ratio between patients in the sitting and supine position. For patients who had undergone both a sitting and supine lumbar spine MRI, the psoas anatomy was compared using a paired $t$-test. For patients who had undergone only a sitting or supine MRI, a propensity score match (PSM), a statistical analysis of observational data, was used to estimate the effect of covariates that predict receiving the treatment. The PSM was performed to match patients for age, BMI, and gender. 

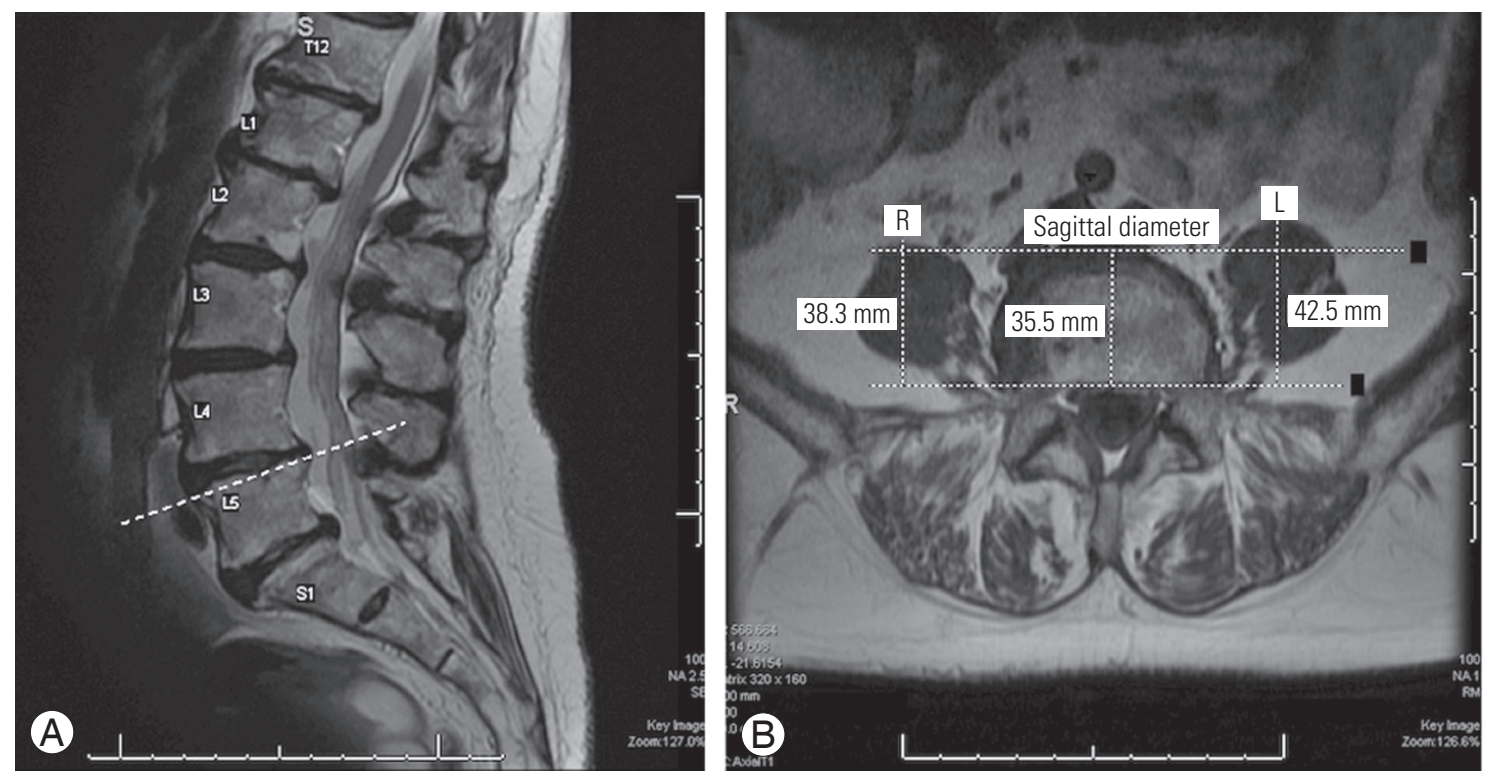

Fig. 1. (A, B) Sagittal view of the lumbar spine with scout line corresponding to the axial view of the $L 4-L 5$ superior endplate.

Table 1. Percentage increase the psoas:disc ratio in sitting versus supine MRI at each respective intervertebral disc level

\begin{tabular}{|c|c|c|c|c|c|c|c|c|c|}
\hline \multirow[b]{2}{*}{ Variable } & \multirow{2}{*}{$\begin{array}{l}\text { Disc } \\
\text { level }\end{array}$} & \multicolumn{4}{|c|}{ Left psoas } & \multicolumn{4}{|c|}{ Right psoas } \\
\hline & & Sitting & Supine & $\begin{array}{c}\% \text { Increase in } \\
\text { sitting }\end{array}$ & $p$-value & Sitting & Supine & $\begin{array}{c}\% \text { Increase in } \\
\text { sitting }\end{array}$ & $p$-value \\
\hline \multicolumn{10}{|c|}{ Propensity matched } \\
\hline & $\mathrm{L} 1-\mathrm{L} 2$ & 0.770 & 0.701 & 9.843 & 0.133 & 0.710 & 0.732 & -3.005 & 0.681 \\
\hline & L2-L3 & 1.020 & 0.915 & 11.475 & 0.010 & 1.023 & 0.860 & 18.953 & $<0.001$ \\
\hline & L3-L4 & 1.210 & 1.034 & 17.021 & $<0.001$ & 1.232 & 1.005 & 22.587 & $<0.001$ \\
\hline & L4-L5 & 1.535 & 1.283 & 19.641 & $<0.001$ & 1.581 & 1.281 & 23.419 & $<0.001$ \\
\hline \multicolumn{10}{|c|}{ BOTH $^{\mathrm{a})}$ group } \\
\hline & $\mathrm{L} 1-\mathrm{L} 2$ & 0.798 & 0.639 & 24.883 & 0.040 & 0.725 & 0.692 & 4.769 & 0.642 \\
\hline & $\mathrm{L} 2-\mathrm{L} 3$ & 1.019 & 0.864 & 17.940 & 0.005 & 1.019 & 0.868 & 17.396 & $<0.0001$ \\
\hline & L3-L4 & 1.238 & 0.999 & 23.924 & 0.004 & 1.272 & 0.973 & 30.730 & $<0.0001$ \\
\hline & L4-L5 & 1.660 & 1.250 & 32.800 & 0.004 & 1.680 & 1.226 & 37.031 & $<0.0001$ \\
\hline
\end{tabular}

Left versus right psoas in propensity score matched and BOTH groups.

$\mathrm{MRI}$, magnetic resonance imaging.

${ }^{\text {a) }}$ atients had undergone both sitting and supine MRI.

\section{Results}

\section{Demographics}

A total of 269 patients had undergone lumbar spine MRI over the study period. Of these, 60 patients had sitting MRIs and 209 patients had supine MRIs, of those with sitting MRIs, 30 were male and 30 were female and of those with supine MRIs, 84 were male and 125 were female. Thirteen of the total 269 patients had both supine and sitting MRIs, with an equal amount of males and females.

\section{Outcomes}

When comparing the psoas morphology in patients with both sitting and supine MRIs, there was an increased 
psoas:disc ratio in the sitting position. Moreover, in the propensity matched cohorts, patients with sitting MRIs also demonstrated an increased psoas:disc ratio than those with supine MRIs. The ratio was found to increase at the more caudal intervertebral discs. Furthermore, the difference in the psoas:disc ratio between sitting and supine increased in the more caudal intervertebral disc levels (Table 1, Fig. 2).

\section{Propensity score matched group}

Within the 269 patient MRIs evaluated, the patients with both supine and sitting MRIs were classified into the "BOTH" group. For the two separate groups of patients with either a supine MRI or a sitting MRI a PSM was then performed to match for age, BMI, and gender to produce two groups of 43 patients. In Table 2, the PSM and BOTH groups were separated and results were collected for each at each individual disc level. The left and right aspects of the psoas muscle were also measured separately. At the L1-L2 disc there was no statistically significant change between sitting and supine. At the L2-L3 disc there was an $11.5 \%$ increase $(p=0.01)$ in psoas:disc ratio on the left and $18.9 \%$ increase on the right psoas $(p<0.001)$ in the sitting patients. At the $\mathrm{L} 3-\mathrm{L} 4$ disc there was a $17 \%$ increased psoas:disc ratio on the left and $22.6 \%$ on the right psoas $(p<0.001$ for both). At the L4-L5 disc there was a $19.6 \%$ increase in the psoas:disc ratio on the left and $23.4 \%$ on the right psoas ( $p<0.001$ for both).

\section{Patients with BOTH sitting and supine magnetic resonance imaging}

For the group "BOTH", patients that underwent both sitting and supine MRIs, at the L1-L2 disc there was a $24.8 \%$ increase ( $p=0.04)$ in psoas:disc ratio on the left and no

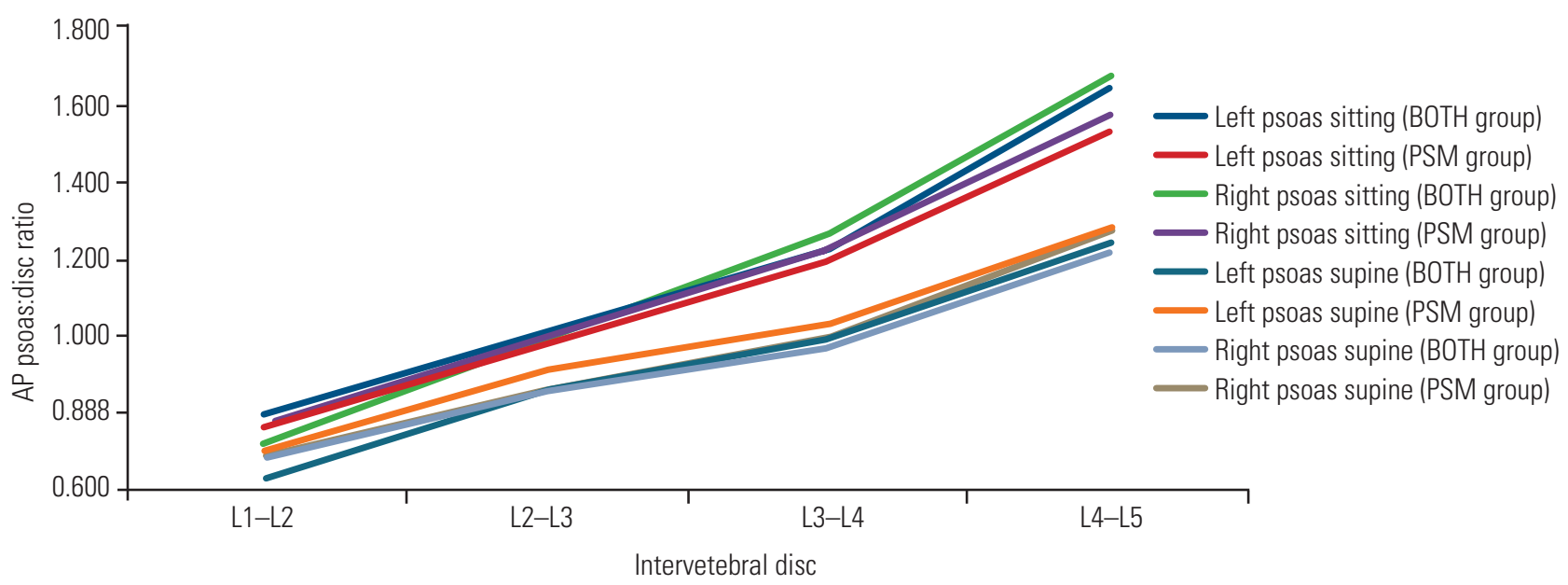

Fig. 2. Graphical representation of the increase in psoas:disc ratio from the $\mathrm{L} 1-\mathrm{L} 2$ disc to the $\mathrm{L} 4-\mathrm{L} 5$ disc bilaterally on sitting and supine MRIs. Note the increased difference in AP psoas:disc ratio between the sitting and supine groups at more cephalad discs in the BOTH ${ }^{\text {al }}$ group and the PSM group. MRI, magnetic resonance imaging; AP, anteroposterior; PSM, propensity score match. ${ }^{\text {la }}$ Patients had undergone both sitting and supine MRIs.

Table 2. Differences between the BOTH ${ }^{\mathrm{a}}$, supine and sitting patient groups with regards to age, BMl, and gender

\begin{tabular}{|c|c|c|c|c|c|c|}
\hline \multirow{2}{*}{ Variable } & \multirow{2}{*}{ Both } & \multicolumn{2}{|c|}{ Supine } & \multicolumn{2}{|c|}{ Sitting } & \multirow{2}{*}{$p$-value sitting vs. supin } \\
\hline & & Mean & SD & Mean & SD & \\
\hline Age (yr) & 58.77 & 57.98 & 14.882 & 55.46 & 15.996 & 0.3015 \\
\hline BMI $\left(\mathrm{kg} / \mathrm{m}^{2}\right)$ & 28.6654 & 27.3864 & 5.39388 & 29.7579 & 6.31889 & 0.0090 \\
\hline Gender & 7F:6M & 115F:80M & 0.493 & $24 \mathrm{~F}: 24 \mathrm{M}$ & 0.505 & 0.2606 \\
\hline
\end{tabular}

Comparison of propensity score matched group and BOTH group factors.

BMI, body mass index; SD, standard deviation; F, female; M, male.

${ }^{a}$ Patients had undergone both sitting and supine magnetic resonance imaging. 
statistically significant change on the right side. At the L2L3 disc there was a $17.9 \%$ increase $(p=0.005)$ in psoas:disc ratio on the left and $17.4 \%$ increase on the right psoas $(p<0.0001)$ in the sitting patients. At the L3-L4 disc there was a $23.9 \%$ increase $(p=0.004)$ in psoas:disc ratio on the left and a $30.7 \%$ increase on the right psoas $(p<0.0001)$ in the sitting patients. At the L4-L5 disc there was a $32.8 \%$ increase $(p=0.004)$ increase in psoas:disc ratio on the left and $37 \%$ increase on the right psoas $(p<0.0001)$ in the sitting patients (Figs. 3, 4). Additionally, there was an observed $137 \%$ maximal increase in the sitting L4-L5 left psoas muscle AP diameter versus the supine and an observed 97\% maximal increase in the sitting L4-L5 right psoas muscle AP diameter versus the supine.

\section{Discussion}

Preoperative analysis of MRI images of the lumbar spine allows surgeons to observe lumbar spine bony and neuroanatomy, the location of vascular structures and the psoas morphology. Such analysis helps the surgeon to decide the feasibility of various operative approaches for lumbar fu-
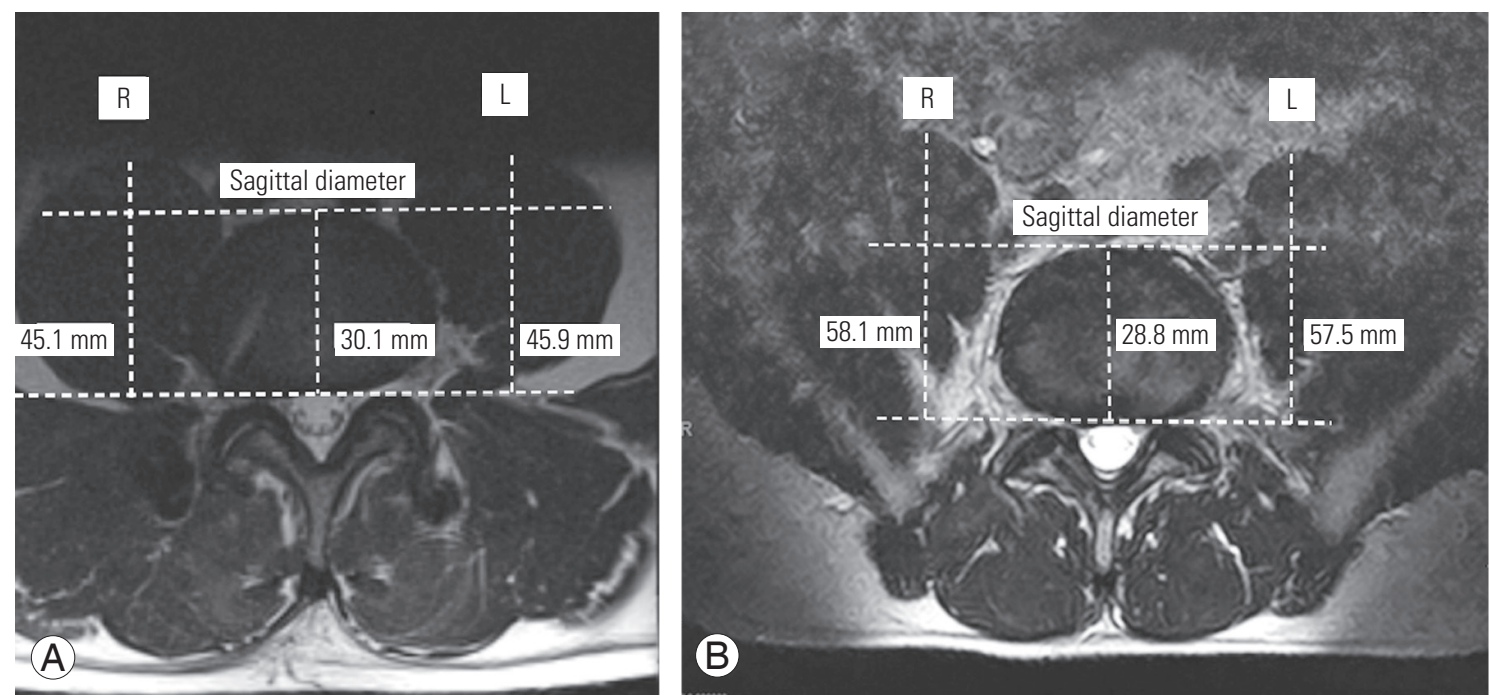

Fig. 3. T2-weighted axial magnetic resonance imaging images at $L 4-L 5$ of patient 1 in the supine (A) and sitting (B) positions demonstrating psoas morphology change, characterized by an increase in anterior-posterior psoas:disc ratio and decrease in mediallateral diameter in sitting.
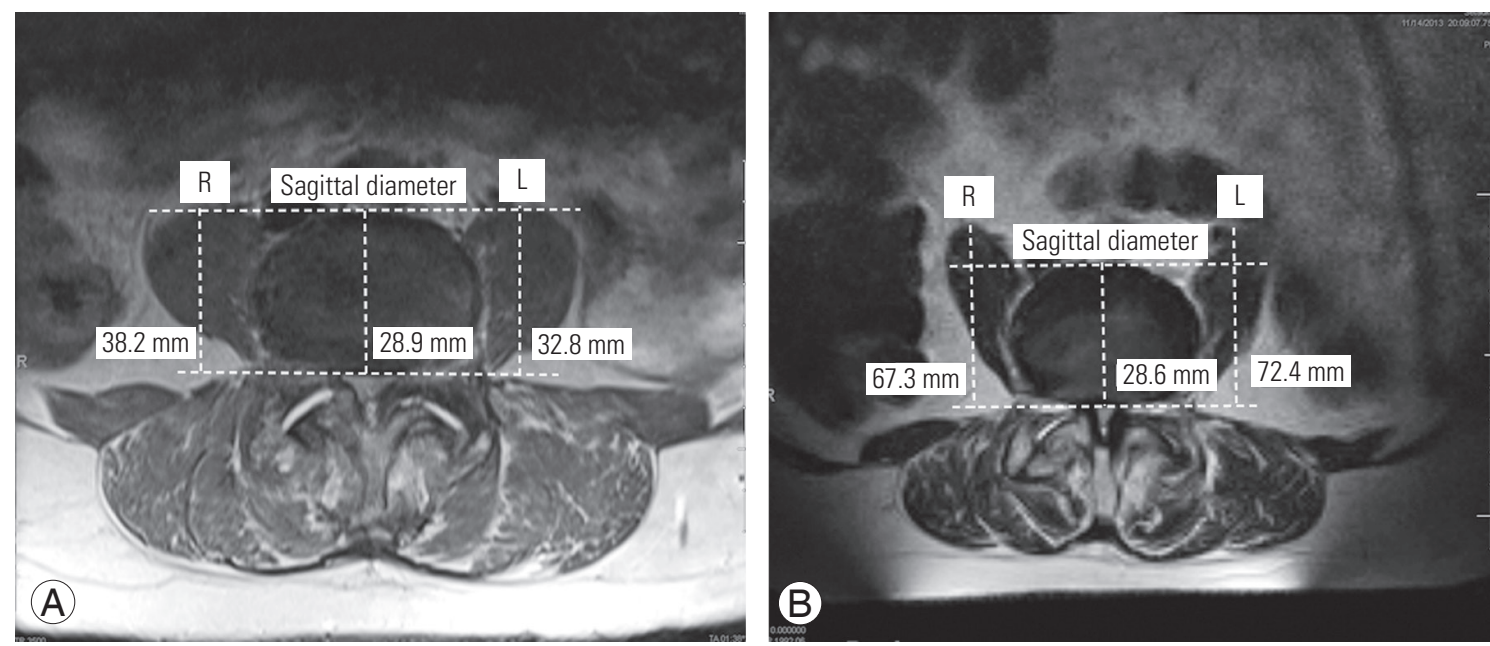

Fig. 4. T2-weighted axial magnetic resonance imaging images at $L 4-L 5$ of patient 2 in the supine $(A)$ and sitting (B) positions demonstrating psoas morphology change, characterized by an increase in anterior-posterior psoas:disc ratio and decrease in mediallateral diameter in sitting. 
sion surgeries. With regard to psoas anatomy, the current study establishes that patients in the sitting position have a more anterior displacement of the psoas muscle. This finding may affect a surgeon's decision to proceed with a lateral approach if the psoas and the lumbar plexus shift anterior to the corridor used to perform the lateral discectomy and fusion.

Access to the lumbar spine in the safest, most efficient and minimally invasive way is an important objective for lumbar spine surgery. Minimally invasive spine surgery theoretically leads to less blood loss and tissue trauma [5] and reduces recovery time [4]. The trans-psoas approach and the oblique anterior-to-psoas approach, while providing a less invasive access to the intervertebral disc, have been associated with injury to the lumbar plexus during the procedure, which can occur during the penetration and retraction of the psoas muscle [4]. The difficulty lies in how to successfully determine a point of entry to split the psoas muscle [11].

Guerin et al. [11] analyzed the location of retroperitoneal vessels and the nerve roots in the retroperitoneal space relative the intervertebral disc spaces using MRIs and determined safe zones that would avoid nerve and vessel injuries during a procedural approach. The safe working zone was defined as the anterior passage between vessels and lumbar plexus and was measured as a percentage of the sagittal vertebral body diameter from anterior to posterior. They reported the safe working zone as being, $75.3 \%$ at $\mathrm{L} 1-\mathrm{L} 2,59.5 \%$ at $\mathrm{L} 2-\mathrm{L} 3,51.9 \%$ at $\mathrm{L} 3-\mathrm{L} 4$, and $37.8 \%$ at L4-L5 levels [11].

Furthermore, Spivak et al. [9] determined in their cadaveric anatomic study of 12 cadavers (24 psoas muscles) to delineate the safe zone for retractor placement that no nerve root encroached anteriorly beyond $33 \%$ of the intervertebral disc. These measurements were made via the AP excursion of each of the L2, L3, L4 nerve roots from the posterior border of the vertebral body to its most anterior location, which were taken with a caliper [9]. They also reported that the mean AP psoas-vertebral body coverage increased significantly from L2-L3 to L3L4 $(79.2 \% \pm 10.2 \%$ versus $86.6 \% \pm 6.1 \%)$ but did not differ significantly from L3-L4 to L4-L5 $(86.6 \% \pm 6.1 \%$ versus $84.8 \% \pm 6.8 \%)$ or between the left and right sides or between males and females [9]. All of the lumbar nerve roots were within the posterior half of the disc space. They recommended that the lumbar plexus need not be directly visualized via the lateral trans-psoas approach but neuro-monitoring during the muscle dissection, but neural stimulation with EMG and retractor docked anterior to the midpoint of the disc on lateral fluoroscopy was recommended [9].

The psoas major increases in size as it descends the trunk, moving anteriorly and slightly laterally in relation to the vertebral body [12]. The psoas major's trend of increasing size continues to the level of L4-L5 where its cross-sectional area then decreases to the S1 level [12]. Furthermore, the size of the left and right psoas major muscles are symmetrical, where paired $t$-test showed no significant left and right difference in psoas cross section area in a MRI study conducted by Reid et al. [12]. When the lumbar spine is undergoing flexion, without hip flexion, the upper fascicles of the psoas major shorten by 5-14 $\mathrm{mm}$ while the lower fascicles show little change in length [13]. The moment arms of the fascicles increase in magnitude in a positive sense, the flexion moment arms become larger and extension moment arms become smaller or convert to flexion moment arms [13].

Davis et al. [14] conducted a cadaveric study which focused upon the retroperitoneal oblique passage to the L2 to S1 discs and their study determined that the use of this particular approach, anterior to the psoas muscle, may avoid many of the anatomic structure-associated complication with the anterior or trans-psoas approaches, but lumbar plexus injury is still a major risk factor when considering this approach, as the nerve roots may still be compressed against the transverse process with retraction [8].

Regev et al. [15] performed a morphometric study using MRI exams to determine the anatomic position for the nerve roots and large retroperitoneal vessels in relation to the vertebral body. They determined that the risk of injury to the ventral nerve roots and retroperitoneal large vessels increased significantly at the L4-L5 level [15], consistent to the reported results by Guerin et al. [11]. The study conducted by Regev et al. [15] used radiographic reference points and the relative ratio between the anatomical structures and the vertebral body for radiographic measurement similar to our own study and results.

Our results established that sitting (i.e., hip flexion) results in anterior displacement of the psoas in the BOTH patient group, and in the propensity matched cohorts. The difference was similar in matched patients and with propensity score matching analyses; however, the number of patients in the "BOTH" group was lower. These results 
show that when considering the trans-psoas and oblique approaches, sitting MRIs may dissuade surgeons from attempting an LLIF in specific patients. Flexion of the hips to 45-60 degrees intraoperatively has been advocated by O'Brien et al. [16] in which they reported it would reduce strain in the lumbar plexus at the L4-L5 disc. This cadaveric study did not simulate lateral retractor placement in the psoas; however, the cadaveric lower extremity had been amputated mid-thigh. The effect of hip and knee flexion/extension with lateral retractor placement remains unknown; however, our data suggests that hip flexion may translate the lumbar plexus further anteriorly, therefore increasing the risk of docking onto-, or posterior to the lumbar plexus and causing inadvertent plexus injury during surgical approach or retraction.

A limitation of this study is that it does not directly comment upon the position of the lumbar plexus in sitting and supine positions. Accurate assessment of the plexus could not be performed on these routine investigations owing to limited lumbar plexus visualization due to the oblique plane of the nerve path on the axial and sagittal images. Additionally, although the fat streak can be used as a surrogate for where the lumbar plexus is, its accuracy has not been validated, so we opted to use the AP dimensions of the psoas as the surrogate. We cannot therefore specifically make recommendations regarding intra-operative positioning for the patient; however, we suggest that standardization in lumbar MRI image acquisition is an important step in further understanding psoas and lumbar plexus anatomy in surgical planning. We had a limited number of patients with both sitting and supine lumbar MRIs (13 patients); however despite this, a significant change in psoas morphology was still demonstrable.

\section{Conclusions}

Anteroposterior psoas geometry is altered between sitting and supine posture on MRI with implications in planning trans-psoas approaches for lateral lumbar interbody fusion. The psoas and therefore lumbar plexus is anteriorly displaced in sitting MRI, by increasing magnitude at more caudal discs. The posture in which the lumbar MRI is performed may have implications on selection of patients for lateral approach and the hip position for a safe working corridor intraoperatively.

\section{Conflict of Interest}

No potential conflict of interest relevant to this article was reported.

\section{References}

1. Regev GJ, Kim CW, Tomiya A, et al. Psoas muscle architectural design, in vivo sarcomere length range, and passive tensile properties support its role as a lumbar spine stabilizer. Spine (Phila Pa 1976) 2011;36:E1666-74

2. Jorgensson A. The iliopsoas muscle and the lumbar spine. Aust J Physiother 1993;39:125-32.

3. Banagan K, Gelb D, Poelstra K, Ludwig S. Anatomic mapping of lumbar nerve roots during a direct lateral transpsoas approach to the spine: a cadaveric study. Spine (Phila Pa 1976) 2011;36:E687-91.

4. Guérin P, Obeid I, Bourghli A, et al. The lumbosacral plexus: anatomic considerations for minimally invasive retroperitoneal transpsoas approach. Surg Radiol Anat 2012;34:151-7.

5. Anand N, Baron EM, Thaiyananthan G, Khalsa K, Goldstein TB. Minimally invasive multilevel percutaneous correction and fusion for adult lumbar degenerative scoliosis: a technique and feasibility study. J Spinal Disord Tech 2008;21:459-67.

6. Ozgur BM, Aryan HE, Pimenta L, Taylor WR. Extreme lateral interbody fusion (XLIF): a novel surgical technique for anterior lumbar interbody fusion. Spine J 2006;6:435-43.

7. Mayer HM. A new microsurgical technique for minimally invasive anterior lumbar interbody fusion. Spine (Phila Pa 1976) 1997;22:691-9.

8. Mehren C, Mayer HM, Zandanell C, Siepe CJ, Korge A. The oblique anterolateral approach to the lumbar spine provides access to the lumbar spine with few early complications. Clin Orthop Relat Res 2016;474:2020-7.

9. Spivak JM, Paulino CB, Patel A, Shanti N, Pathare N. Safe zone for retractor placement to the lumbar spine via the transpsoas approach. J Orthop Surg (Hong Kong) 2013;21:77-81.

10. He L, Kang Z, Tang WJ, Rong LM. A MRI study of lumbar plexus with respect to the lateral transpsoas approach to the lumbar spine. Eur Spine J 2015;24:2538-45. 
11. Guerin P, Obeid I, Gille O, et al. Safe working zones using the minimally invasive lateral retroperitoneal transpsoas approach: a morphometric study. Surg Radiol Anat 2011;33:665-71.

12. Reid JG, Livingston LA, Pearsall DJ. The geometry of the psoas muscle as determined by magnetic resonance imaging. Arch Phys Med Rehabil 1994;75:7038.

13. Bogduk N, Pearcy M, Hadfield G. Anatomy and biomechanics of psoas major. Clin Biomech (Bristol, Avon) 1992;7:109-19.

14. Davis TT, Hynes RA, Fung DA, et al. Retroperitoneal oblique corridor to the L2-S1 intervertebral discs in the lateral position: an anatomic study. J Neurosurg Spine 2014;21:785-93.

15. Regev GJ, Chen L, Dhawan M, Lee YP, Garfin SR, Kim CW. Morphometric analysis of the ventral nerve roots and retroperitoneal vessels with respect to the minimally invasive lateral approach in normal and deformed spines. Spine (Phila Pa 1976) 2009;34:13305.

16. O’Brien J, Haines C, Dooley ZA, Turner AW, Jackson D. Femoral nerve strain at L4-L5 is minimized by hip flexion and increased by table break when performing lateral interbody fusion. Spine (Phila $\mathrm{Pa} 1976$ ) 2014;39:33-8. 